\title{
Antithrombotic therapy in patients with atrial fibrillation undergoing percutaneous coronary intervention, including compliance with current guidelines - data from the POLish Atrial Fibrillation (POL-AF) Registry
}

\author{
Beata Uziębło-Życzkowska ${ }^{1}$, Paweł Krzesiński ${ }^{1}$, Małgorzata Maciorowska ${ }^{1}$, Iwona Gorczyca ${ }^{2,3}$, \\ Olga Jelonek $^{2,3}$, Maciej Wójcik ${ }^{4}$, Robert Błaszczyk ${ }^{4}$, Agnieszka Kapłon-Cieślicka ${ }^{5}$, Monika Gawałko ${ }^{5}$, \\ Tomasz Tokarek ${ }^{6}$, Renata Rajtar-Salwa ${ }^{6}$, Jacek Bil ${ }^{7}$, Michał Wojewódzki ${ }^{7}$, Anna Szpotowicz ${ }^{8}$, \\ Małgorzata Krzciuk ${ }^{8}$, Janusz Bednarski ${ }^{9}$, Elwira Bakuła-Ostalska ${ }^{9}$, Anna Tomaszuk-Kazberuk ${ }^{10}$, Anna \\ Szyszkowska $^{11}$, Marcin Wełnicki ${ }^{12}$, Artur Mamcarz ${ }^{12}$, Beata Wożakowska-Kapłon ${ }^{2,3}$ \\ ${ }^{1}$ Department of Cardiology and Internal Diseases, Military Institute of Medicine, Warsaw, Poland; ${ }^{2} 1$ st Clinic of Cardiology and Electrotherapy, \\ Swietokrzyskie Cardiology Centre, Kielce, Poland; ${ }^{3}$ Collegium Medicum, The Jan Kochanowski University, Kielce, Poland; ${ }^{4}$ Department of \\ Cardiology, Medical University of Lublin, Lublin, Poland; ${ }^{5} 1$ st Chair and Department of Cardiology, Medical University of Warsaw, Warsaw, \\ Poland; ${ }^{6}$ Department of Cardiology and Cardiovascular Interventions, University Hospital, Krakow, Poland; ${ }^{7}$ Department of Invasive Cardiology, \\ Centre of Postgraduate Medical Education, Central Clinical Hospital of the Ministry of Interior and Administration, Warsaw, Poland; ${ }^{8}$ Department \\ of Cardiology, Regional Hospital, Ostrowiec Świętokrzyski, Poland; ${ }^{9}$ Department of Cardiology, St John Paul II Western Hospital, Grodzisk \\ Mazowiecki, Poland; ${ }^{10}$ Department of Cardiology, Medical University of Bialystok, Białystok, Poland; ${ }^{11}$ Department of Cardiology, University \\ Hospital of Bialystok, Białystok, Poland; ${ }^{12} 3$ rd Department of Internal Diseases and Cardiology, Warsaw Medical University, Warsaw, Poland \\ Contributions: (I) Conception and design: B Uziębło-Życzkowska, P Krzesiński, I Gorczyca, A Kapłon-Cieślicka, B Wożakowska-Kapłon; (II) \\ Administrative support: P Krzesiński, B Wożakowska-Kapłon; (III) Provision of study materials or patients: All authors; (IV) Collection and assembly \\ of data: All authors; (V) Data analysis and interpretation: B Uziębło-Życzkowska, P Krzesiński; (VI) Manuscript writing: All authors; (VII) Final \\ approval of manuscript: All authors. \\ Correspondence to: Iwona Gorczyca, MD, PhD. $1^{\text {st }}$ Clinic of Cardiology and Electrotherapy, Swietokrzyskie Cardiology Centre, 25-736 Kielce, ul. \\ Grunwaldzka 45, Poland. Email: iwona.gorczyca@interia.pl.
}

Background: Although triple antithrombotic therapy (TAT) is recommended in patients with atrial fibrillation (AF) undergoing percutaneous coronary intervention (PCI), guidelines allow an option of dual antithrombotic therapy (DAT). This study assesses the everyday practice of 10 cardiology departments in antithrombotic therapy in $\mathrm{AF}$ patients undergoing $\mathrm{PCI}$ and its agreement with current guidelines.

Methods: This analysis included medical data of AF patients enrolled in the prospective, observational registry (The POLish Atrial Fibrillation-POL-AF) that underwent PCI [elective or due to acute coronary syndrome (ACS)].

Results: Of the 3,999 consecutive subjects included, a final analysis was performed on 359 patients that underwent PCI: 148 with urgent PCI due to ACSand 211 patients with elective PCI. Eighty patients in the ACS-group and 120 patients in the elective-PCI group were treated with TAT, although guidelines also allowed DAT. Of 316 patients treated with oral anticoagulants as a part of combination therapy, 275 were on non-vitamin $\mathrm{K}$ antagonist oral anticoagulant (NOAC). Reduced doses of NOAC were used in 74 patients treated with rivaroxaban, 60 patients with dabigatran, and 54 patients with apixaban. The proportion of patients treated with reduced NOAC doses adequately to the guidelines was $29 \%, 100 \%$, and $33 \%$ for rivaroxaban, dabigatran, and apixaban, respectively. Inappropriate low doses of NOACs were used in $71 \%$ of subjects on rivaroxaban and $67 \%$ on apixaban.

Conclusions: In patients with AF undergoing PCI, NOACs are definitely preferred over vitamin-K antagonists (VKAs) in TAT/DAT, and an aggressive antithrombotic strategy with TAT is frequently chosen even if DAT is permissible by the guidelines. Label adherence of using reduced NOAC dose during 
combination therapy is not satisfactory for apixaban and rivaroxaban and probably results from too cautious an approach to the known indications for reduced therapy. The study is registered in the database Clinical Trials-NCT04419012.

Keywords: Acute coronary syndrome (ACS); antithrombotic therapy; atrial fibrillation (AF); percutaneous coronary intervention (PCI)

Submitted Oct 05, 2020. Accepted for publication Nov 16, 2020.

doi: $10.21037 / \mathrm{cdt}-20-839$

View this article at: http://dx.doi.org/10.21037/cdt-20-839

\section{Introduction}

Most patients with atrial fibrillation (AF) require chronic oral anticoagulation (OAC) to prevent thromboembolic events, including stroke. Moreover, almost 20-30\% of them suffer from coronary artery disease (1-3). In consequence, the $\mathrm{AF}$ population accounts for approximately $5 \%$ to $7 \%$ of patients undergoing percutaneous coronary intervention (PCI) (4). For many years, the combination of dual antiplatelet therapy (DAPT) and OAC (triple antithrombotic therapy, TAT) was recommended by the European Society of Cardiology (ESC) in patients with AF undergoing PCI. Recent randomized clinical trials (PIONEER AF-PCI, WOEST, RE-DUAL PCI, ENTRUST AF-PCI, and AUGUSTUS) demonstrated the benefits of OAC and single antiplatelet therapy (dual antithrombotic therapy, DAT), which, with comparable antithrombotic efficacy, reduced the frequency of major bleeding in patients undergoing PCI (5-9). The most recent guidelines step forward by shortening the time of TAT, even if recommended $(10,11)$.

Another important issue is the selection of appropriate OAC doses. In particular, in accordance with the guidelines that recommend the use of non-vitamin $\mathrm{K}$ antagonist oral anticoagulant (NOAC) instead of vitamin-K antagonists (VKAs), the use of NOACs in the treatment of patients with AF has grown rapidly in recent years $(10,11)$. However, the reduction of NOAC doses is recommended in some clinical settings. A recent large meta-analysis showed that in eligible patients, such a treatment strategy, compared to the use of warfarin, was associated with a decreased risk of bleeding and complications such as stroke or systemic embolism (12).

This study assesses the everyday practice of 10 cardiology departments in antithrombotic therapy in a nationwide cohort of hospitalized patients with $\mathrm{AF}$ undergoing elective or urgent PCI and its agreement with current guidelines. Additionally, we estimate how often reduced NOAC doses were used during combination antithrombotic therapy and whether a low NOAC dose was applied according to ESC guidelines.

We present the following article in accordance with the STROBE reporting checklist (available at: http://dx.doi. org/10.21037/cdt-20-839).

\section{Methods}

The study was conducted according to Good Clinical Practice guidelines and the Declaration of Helsinki (as revised in 2013). The study protocol was approved by the Ethics Committee of the Swietokrzyska Medical Chamber in Kielce (104/2018). The Ethics Committee waived the requirement of obtaining informed consent from the patients to participate in the study.

\section{Study design and patients}

Our study was a part of a prospective, observational, multicenter registry based on the medical data of consecutive AF patients who were admitted to Polish cardiology departments between January and December 2019 (The POLish Atrial Fibrillation, POL-AF). Patients were included in the register within two selected weeks of every month (full weeks, seven days), excluding patients scheduled for ablation (usually these patients are younger and do not have concomitant diseases). Patients with AF diagnosed on admission to hospital or during hospitalization, including patients with potentially reversible causes of arrhythmia, were included in the registry. No explicit exclusion criteria were defined to avoid a biased selection of patients and achieve a cohort close to "real life". From the whole group of patients with AF mentioned above, we separated those who underwent PCI during hospitalization [elective PCI or performing due to acute coronary syndrome (ACS)]. 
The collected data included baseline demographic characteristics, the results of a clinical evaluation, laboratory tests, echocardiography, and the treatment strategy recommended upon discharge with a particular emphasis on an antithrombotic treatment strategy. The clinical evaluation focused on age, sex, co-morbidities, type of AF, type and doses of anticoagulants. $\mathrm{CHA}_{2} \mathrm{DS}_{2}$ VASc and HASBLED scores were calculated for each patient according to the recommendations $(13,14)$. Laboratory tests included the evaluation of renal function [estimated glomerular filtration rate (eGFR), creatinine] and morphology parameters. eGFR was calculated from the Modification of Diet in Renal Disease or Chronic Kidney Disease Epidemiology Collaboration formula. The analyzed echocardiographic parameter was the left ventricular ejection fraction (LVEF).

\section{Type of antithrombotic therapy in AF patients undergoing PCI}

An analysis of the antithrombotic therapy was performed in the whole group and separately in a group of AF patients undergoing elective PCI- "the elective-PCI group" and urgent PCI during ACS—“the ACS group."

Recommended antithrombotic therapy was defined as therapy rigorous according to recommendations, which means TAT (DAPT + OAC) or DAT, defined as OAC + single antiplatelet agent, if the patient had a high risk of bleeding complications indicated by HASBLED $\geq 3$ points (13-16).

Other types of treatment were divided into two groups. The first group called "Lenient antithrombotic therapy" was defined as treatment using DAT despite indications for TAT, using only DAPT, using single antithrombotic therapy, and no antithrombotic therapy. The second group called "Aggressive antithrombotic therapy" included those using TAT despite permissible DAT treatment.

\section{Use of NOACs as a part of combined therapy in $A F$ patients undergoing PCI}

An analysis of the application of the OAC type of patients was conducted. The use of reduced NOAC doses during combined treatment in post-PCI patients with AF was specifically evaluated, analyzing the compliance of dose reduction with the ESC guidelines $(10,11,15,16)$ and the Summary of Product Characteristics registered in European Medicine Agency (EMA) (17-19). According these mentioned above documents patients were categorized by the usage of a reduced NOAC dose: appropriate reduced dose, inappropriate low dose, and inappropriate high dose. Approved dose criteria were specific for each NOAC according to the following patient characteristics: weight, age, renal function, and concomitant medications.

\section{Statistical analysis}

The statistical analysis was performed using Statistica 12.0 (StatSoft, Inc., Tulsa, OK, USA). The distribution and normality of the data were assessed by visual inspection and the Kolmogorov-Smirnov test. Continuous variables were presented as means \pm standard deviations (SD) and categorical variables as absolute and relative frequencies (percentages). To analyze the differences between subgroups, the Student's $t$-test for normally distributed data and the Mann-Whitney U-test if the data were not normally distributed were applied. For categorical variables, the chi square test and Fisher exact test were used. A P value of $<0.05$ indicated statistical significance.

\section{Results}

Of the 3,999 POL-AF registry patients, 364 (9.1\%) underwent PCI during hospitalization. Table 1 presents other reasons for the hospitalization of patients with $\mathrm{AF}$. Five patients died before discharge from hospital (4 with ACS). The remaining 359 patients were subjected to the final analysis: 148 (41\%) patients with urgent PCI due to ACS (the ACS group) and 211 (59\%) patients with elective PCI (the elective-PCI group).

The average age of the entire study group was 73.3 years [235 (65.7\%) of men]. The main comorbidities in the study group were heart failure $(246 ; 68.5 \%)$, hypertension (318; $88.6 \%)$, diabetes mellitus (164; $45.7 \%)$, history of myocardial infarction (182; 50.7\%), previous PCI (196; 54.6\%), and chronic kidney disease $(102 ; 28.4 \%)$. Table 2 presents the detailed characteristics of the study group.

The group of patients undergoing PCI due to ACS compared to those with elective-PCI presented more often a history of bleeding, dialysis, or kidney transplantation, decreased eGFR $\left(<30 \mathrm{~mL} / \mathrm{min} / 1.73 \mathrm{~m}^{2}\right)$, and lower hemoglobin level. The proportion of females was higher and the previous history of PCI was lower in this group.

\section{Analysis of antithrombotic treatment strategy in ACS- group vs. elective-PCI group}

TAT was used more frequently in both patient groups 
Table 1 Reasons for hospitalization of AF patients in the POL-AF study

\begin{tabular}{lc}
\hline Reasons for hospitalization of AF patients & All group; $\mathrm{n}=3,999$ \\
\hline Elective cardioversion & $893(22 \%)$ \\
Cardiac implantable electronic device implantation/reimplantation & $360(9 \%)$ \\
Heart failure & $825(20 \%)$ \\
Ablation from reasons other than AF & $210(5 \%)$ \\
Paroxysmal AF without cardioversion & $252(6 \%)$ \\
Elective coronarography/percutaneous coronary intervention & $383(10 \%)$ \\
Acute coronary syndrome & $247(6 \%)$ \\
Other reasons & $829(21 \%)$ \\
\hline
\end{tabular}

AF, atrial fibrillation; POL-AF, Polish-atrial fibrillation study.

than recommended by the guidelines. An analysis of TAT showed that 80 patients in the ACS group and 120 patients in the elective-PCI group were treated with TAT, despite guidelines allowing for treatment reduction to DAT (i.e., aggressive antithrombotic therapy). Lenient antithrombotic therapy was found much less frequently in 31 of the ACS patients and 63 of the elective-PCI patients. Figures 1 and 2 show detailed antithrombotic therapy data for both groups. Figure 3 presents detailed OAC therapy in the study group.

Antithrombotic therapy with OAC and ticagrelor or prasugrel was not reported in our patients. Ticagrelor plus ASA was used in three patients (one in the elective-PCI group and two in the ACS group).

\section{Analysis of the use of NOAC in AF patients undergoing PCI}

In the study group, 41 patients (11.4\%) were treated with VKAs, 11 were diagnosed with valvular AF, and four were treated with NOACs despite the diagnosis of valvular AF.

Table 3 and Figure 4 present detailed results of the analysis regarding the use of NOACs (including reduced doses of them) in the entire group of patients and separately for the two groups.

The analysis shows that patients received reduced doses of NOACs much more frequently than recommended, especially apixaban and rivaroxaban. In total, reduced doses of NOAC were used in $73(73 \%)$ of patients for rivaroxaban and in $54(55 \%)$ of patients for apixaban, but the percentage of inappropriate low doses of NOACs was $52(71 \%)$ for rivaroxaban and $36(67 \%)$ for apixaban. The problem of using inappropriate high doses of NOAC was minor and related mainly to dabigatran. In the case of dabigatran, all patients received a reduced dose of NOACs in agreement with the guidelines. However, $21 \%$ were recommended a full dose of dabigatran despite indications to reduce it.

The subjects treated with lower doses of rivaroxaban/ apixaban were, as a result of EMA guidelines, older, more frequently with chronic kidney disease, and presenting a lower renal filtration rate and lower body mass index. However, the inappropriate low dose group also showed a higher prevalence of ischemic stroke and higher hemoglobin concentration (see Table 4). An exemplary age-dependency analysis proved that an inappropriately low dose of apixaban is frequently used not only in patients $\geq 80$ years (as the only one of minimum two indications to dose reduction) but also in middle-aged (65-79 years) patients (Figure 5).

A comparison of the ACS group and the elective-PCI group showed that the use of reduced NOAC doses was comparable in both groups (83-77\% vs. 104-62\%; $\mathrm{P}=0.2$; see Table 3).

\section{Discussion}

Decisions on how to treat patients with $\mathrm{AF}$ who are undergoing PCI remain challenging. Many patients with AF require NOAC treatment. Moreover, as our recent study showed, the prevalence of left atrial appendage thrombus in AF patients with lower class (IIa) recommendation to anticoagulants was comparable to the higher one (I class) (20). Doctors have to choose between bleeding risk on the one hand and thromboembolic risk on the other. The findings of our real-life registry showed that some AF patients discharged from the hospital after 
Table 2 The detailed characteristics of the study group

\begin{tabular}{|c|c|c|c|c|c|}
\hline Characteristics & $\mathrm{n}$ & All group; $n=359$ & ACS group; $n=148$ & Elective- $\mathrm{PCl}$ group; $\mathrm{n}=211$ & $\mathrm{P}$ \\
\hline Age, years, mean (SD) & 359 & $73.3(9.5)$ & $74.4(10.5)$ & $72.8(8.7)$ & 0.114 \\
\hline Female sex, n (\%) & 359 & $123(34.3)$ & $60(40.5)$ & $63(29.9)$ & 0.036 \\
\hline Body mass (kg); mean (SD) & 284 & $83.5(17.9)$ & $81.2(18.9)$ & $85.3(16.7)$ & 0.052 \\
\hline \multicolumn{6}{|l|}{ Clinical data; n (\%) } \\
\hline Heart failure & 359 & $246(68.5)$ & $94(63.5)$ & $152(72.0)$ & 0.087 \\
\hline HFrEF & 359 & $119(48.4)$ & $50(34.2)$ & $69(33.5)$ & 0.866 \\
\hline Valvular AF & 359 & $16(4.5)$ & $4(2.7)$ & $12(5.7)$ & 0.177 \\
\hline Hemorrhagic stroke & 359 & $4(1.1)$ & $2(1.35)$ & $2(0.95)$ & 0.72 \\
\hline Ischemic stroke & 359 & $41(11.4)$ & $18(12.2)$ & $23(10.9)$ & 0.711 \\
\hline Peripheral embolism & 359 & $3(0.8)$ & $2(1.35)$ & $1(0.5)$ & 0.473 \\
\hline History of myocardial infarction & 359 & $182(50.7)$ & $71(48)$ & $111(52.6)$ & 0.387 \\
\hline History of CABG & 359 & $36(10.1)$ & $20(13.5)$ & $16(7.6)$ & 0.066 \\
\hline Previous $\mathrm{PCl}$ & 359 & $196(54.6)$ & $64(43.2)$ & $132(62.6)$ & 0.0003 \\
\hline Previous bleeding (including haemorrhagic stroke) & 359 & $12(3.3)$ & $9(6.1)$ & $3(1.4)$ & 0.016 \\
\hline History of gastrointestinal bleeding & 359 & $10(2.8)$ & $5(3.4)$ & $5(2.4)$ & 0.567 \\
\hline $\begin{array}{l}\text { Malignant neoplasm (active/treatment completed } \\
<1 \text { year) }\end{array}$ & 359 & $14(3.9)$ & $6(4.1)$ & $8(3.8)$ & 0.9 \\
\hline Thrombophilia & 359 & $1(0.3)$ & $0(0)$ & $1(0.5)$ & 0.402 \\
\hline Chronic obstructive pulmonary disease & 359 & $36(10.1)$ & $11(7.4)$ & $25(11.8)$ & 0.17 \\
\hline Asthma & 359 & $6(1.7)$ & $1(0.7)$ & $5(2.4)$ & 0.218 \\
\hline Hypothyroidism & 359 & $37(10.3)$ & $12(8.1)$ & $25(11.8)$ & 0.251 \\
\hline Hyperthyroidism & 359 & $18(5.0)$ & $11(7.4)$ & $7(3.3)$ & 0.786 \\
\hline Hypertension & 359 & $318(88.6)$ & $124(83.8)$ & $194(91.9)$ & 0.167 \\
\hline Chronic liver disease & 359 & $11(3.1)$ & $2(1.3)$ & $9(4.3)$ & 0.115 \\
\hline Alcohol abuse & 330 & $14(4.24)$ & $3(2.0)$ & $11(5.6)$ & 0.13 \\
\hline
\end{tabular}

Table 2 (continued) 
Table 2 (continued)

\begin{tabular}{|c|c|c|c|c|c|}
\hline Characteristics & $\mathrm{n}$ & All group; $n=359$ & ACS group; $n=148$ & Elective-PCI group; $\mathrm{n}=211$ & $\mathrm{P}$ \\
\hline HASBLED $\geq 3$ points, $\mathrm{n}(\%)$ & 359 & $275(76.6)$ & $114(77.0)$ & $161(76.3)$ & 0.873 \\
\hline CHA2DS2-VASc, mean (SD) & 385 & $5.05(1.2)$ & $5.02(1.2)$ & 0.648 & 359 \\
\hline \multicolumn{6}{|l|}{ Laboratory data, mean (SD) } \\
\hline Creatinine $(\mathrm{mg} / \mathrm{dL})$ & & & & & 354 \\
\hline eGFR $\left(\mathrm{mL} / \mathrm{min} / 1.73 \mathrm{~m}^{2}\right)$ & 349 & $63.1(23.0)$ & $64.4(26.1)$ & $62.2(20.6)$ & 0.857 \\
\hline eGFR <15, n (\%) & 349 & $6(1.7)$ & $5(3.5)$ & $1(0.5)$ & 0.035 \\
\hline eGFR <30, n (\%) & 349 & $20(5.7)$ & $13(9)$ & $7(3.4)$ & 0.026 \\
\hline Left ventricular ejection fraction (\%) & 299 & $44.3(12.4)$ & $43.5(11.7)$ & $45.4(12.7)$ & 0.173 \\
\hline \multicolumn{6}{|l|}{ Echocardiography data, mean (SD) } \\
\hline Left ventricular ejection fraction (\%) & 299 & $44.3(12.4)$ & $43.5(11.7)$ & $45.4(12.7)$ & 0.173 \\
\hline
\end{tabular}

AF, atrial fibrillation; ACS, acute coronary syndrome; CABG, coronary artery bypass graft; eGFR, estimated glomerular filtration rate; $\mathrm{HFrEF}$, heart failure with reduced ejection fraction; $\mathrm{PCI}$, percutaneous coronary intervention; TIA, transient ischemic attack.

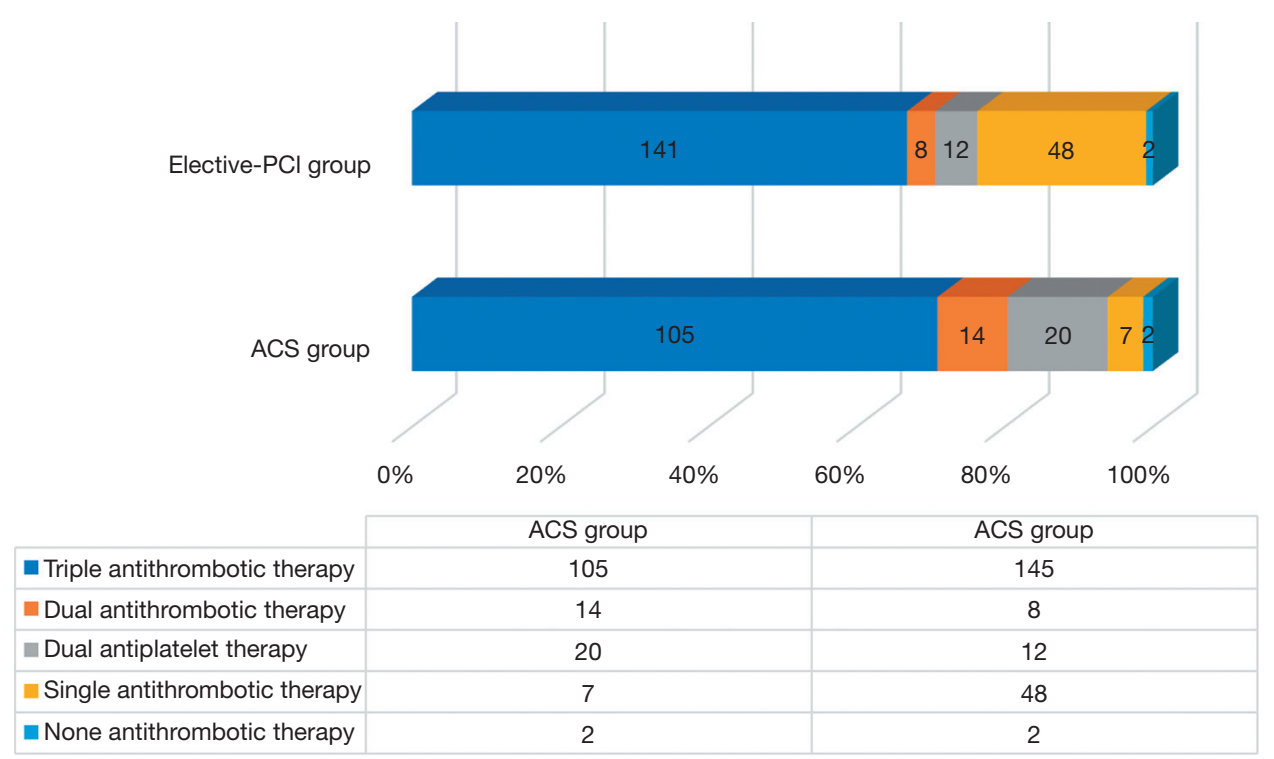

Figure 1 The type of the antithrombotic therapy in AF patients undergoing PCI (Triple antithrombotic therapy: OAC+ASA+clopidogrel/ ticagrelor; Dual antithrombotic therapy: OAC+ASA/clopidogrel/ticagrelor; Dual antiplatelet therapy: ASA+ clopidogrel/ticagrelor; Single antithrombotic therapy: ASA, or clopidogrel, or ticagrelor, or OAC, or heparine). 


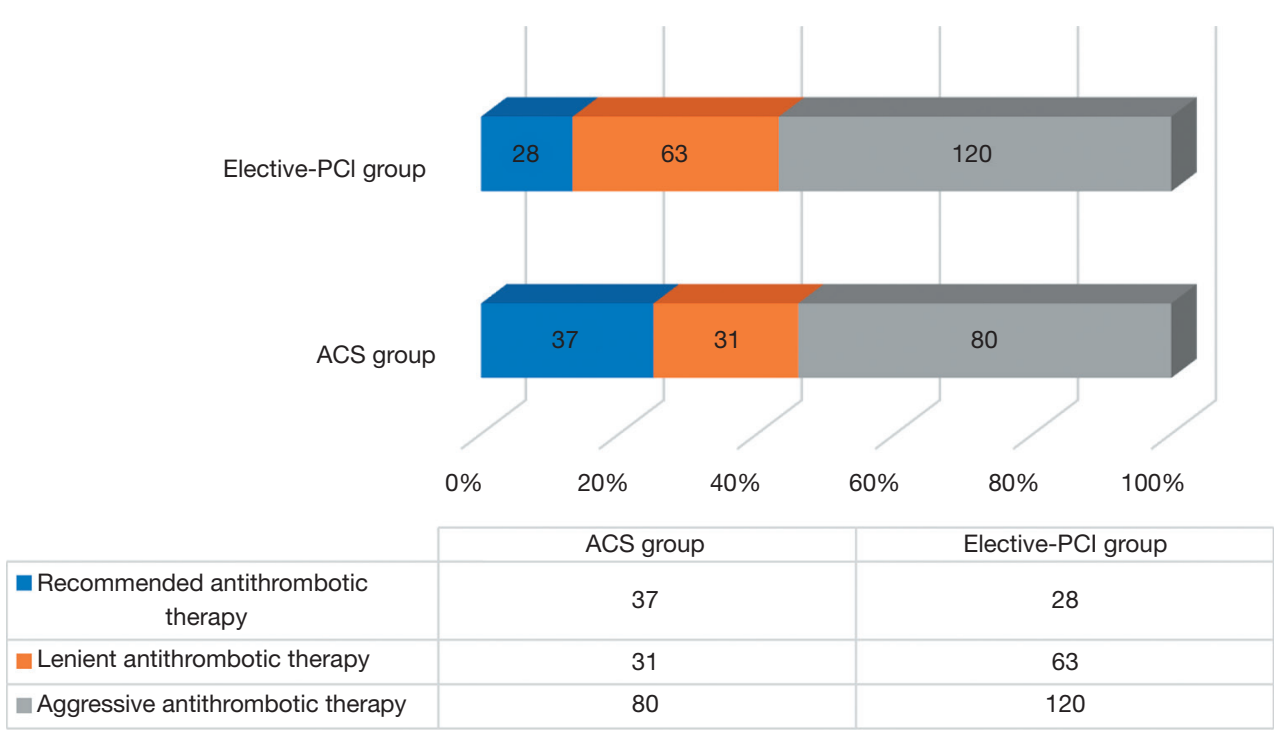

Figure 2 Compliance of the antithrombotic therapy in AF patients undergoing PCI with the guidelines (the terms used are explained in detail in the main text of manuscript). AF, atrial fibrillation; PCI, percutaneous coronary intervention.

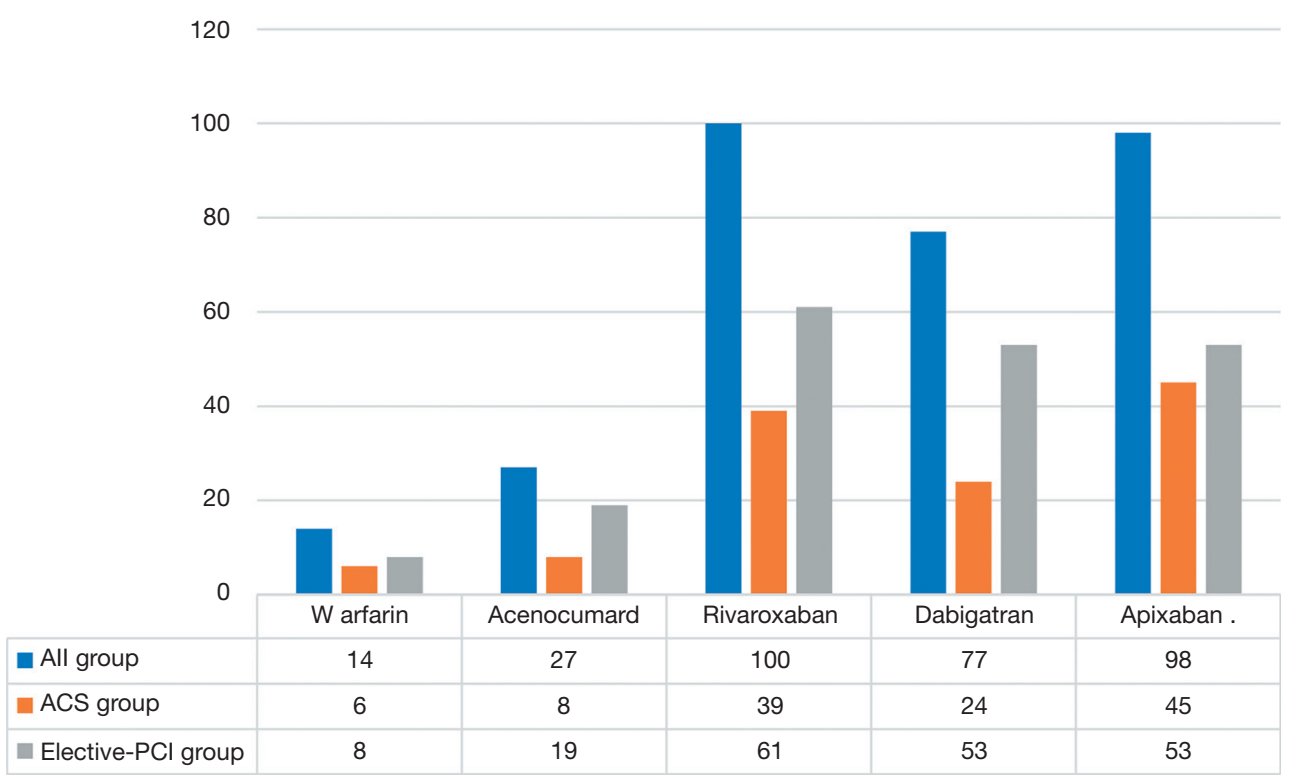

Figure 3 Detailed oral anticoagulation therapy in the study group.

a PCI procedure receive TAT despite an increased risk of bleeding. The study by Bogacki et al. (21) indicated that TAT is associated with high bleeding rates and mortality in those patients. These complications were observed after a relatively short period. Similarly, other authors in a metaanalysis of six studies and comparing the use of TAT $v s$. DAT confirmed that reducing treatment from TAT to
DAT leads to a reduced bleeding risk without affecting the incidence of thromboembolic events (22). In our registry, 80 patients in the ACS-group and 120 patients in the electivePCI group were treated too aggressively with TAT despite guidelines allowing their treatment with DAT. Recent evidence from randomized clinical trials showed (5-9) that DAT seems to be the optimal balance between protecting 
Table 3 Detailed oral anticoagulation therapy data in both groups of patients

\begin{tabular}{|c|c|c|c|c|}
\hline Variables & All group; $n=359$ & ACS group; $n=148$ & Elective-PCI group; $\mathrm{n}=211$ & $P$ \\
\hline VKAs & $41(13 \%)$ & $14(11 \%)$ & $27(14 \%)$ & 0.327 \\
\hline Warfarin & $14(34 \%)$ & $6(43 \%)$ & $8(30 \%)$ & 0.899 \\
\hline Acenocumarol & $27(66 \%)$ & $8(57 \%)$ & $19(70 \%)$ & 0.203 \\
\hline Reduced dose of NOACs & $187(68 \%)$ & $83(77 \%)$ & $104(62 \%)$ & 0.205 \\
\hline Rivaroxaban & $100(36 \%)$ & $39(36 \%)$ & $61(36.5 \%)$ & 0.594 \\
\hline Reduced dose of rivaroxaban & $73(73 \%)$ & $34(87 \%)$ & 39 (64\%) & 0.011 \\
\hline Appropriate reduced dose & $21(29 \%)$ & $8(24 \%)$ & $13(33 \%)$ & 0.924 \\
\hline Reduced dose of dabigatran & $60(78 \%)$ & $23(96 \%)$ & $37(70 \%)$ & 0.108 \\
\hline Appropriate reduced dose & $60(100 \%)$ & $23(100 \%)$ & 37 (100\%) & 0.011 \\
\hline Inappropriate low dose & $0(0 \%)$ & $0(0 \%)$ & $0(0 \%)$ & - \\
\hline Apixaban & $98(36 \%)$ & $45(42 \%)$ & $53(32 \%)$ & 0.268 \\
\hline Reduced dose of apixaban & $54(55 \%)$ & $26(58 \%)$ & $28(53 \%)$ & 0.456 \\
\hline Appropriate reduced dose & $18(33 \%)$ & $12(46 \%)$ & $6(21 \%)$ & 0.051 \\
\hline Inappropriate low dose & $36(67 \%)$ & $14(54 \%)$ & $22(79 \%)$ & 0.288 \\
\hline
\end{tabular}

ACS, acute coronary syndrome; PCI, percutaneous coronary intervention; NOACs, non-vitamin K antagonist oral anticoagulants; VKAs, vitamin $\mathrm{K}$ antagonists.

patients against thromboembolic events and avoiding unnecessary bleeding complications.

In our registry, we do not record data on possible bleeding complications in patients treated too aggressively. Therefore, we were not able to verify previous studies $(6,7,21)$ reporting that the use of TAT in AF patients significantly increases the risk of bleeding complications. Additionally, we did not investigate all potentially co-existing high-risk features for ischemic events mentioned in the guidelines (11), such as diffuse multivessel disease, unfavorable coronary anatomy, and complex revascularization. In some cases, it could explain the aggressive approach to antithrombotic treatment. However, the observed high percentage of aggressively treated patients suggests a significant influence of local practices that should be carefully verified.

Predictably, significantly noticeable in our registry was a trend of using NOACs instead of VKAs. In the present study, nearly $90 \%$ of patients treated with OAC received NOACs. This trend is common in many countries where the use of NOACs is increasing at the expense of VKAs. A recent large meta-analysis (23) aimed to assess benefits and risks associated with the use of NOACs versus VKAs in $\mathrm{AF}$ patients undergoing PCI, with a particular focus on the combination of antithrombotic therapy. The authors unequivocally showed that combined antithrombotic therapy with NOACs (both as DAT or TAT) is safer than with VKAs with respect to bleeding risk and results in no increase in thromboembolic events. Moreover, the magnitude of the effect was larger when NOACs were used in DAT than TAT. These findings were confirmed in the other meta-analysis, which included more than 10,000 patients (participants of four randomized trials: WOEST, PIONEER AF-PCI, RE-DUAL PCI, and AUGUSTUS) (24). The use of NOACs was associated with less bleeding compared to VKAs plus DAPT. Moreover, the authors suggested that using VKAs plus DAPT should generally be avoided. Further, Chi et al. (25) reported that all combination therapies with NOACs were superior in terms of bleeding and noninferior 


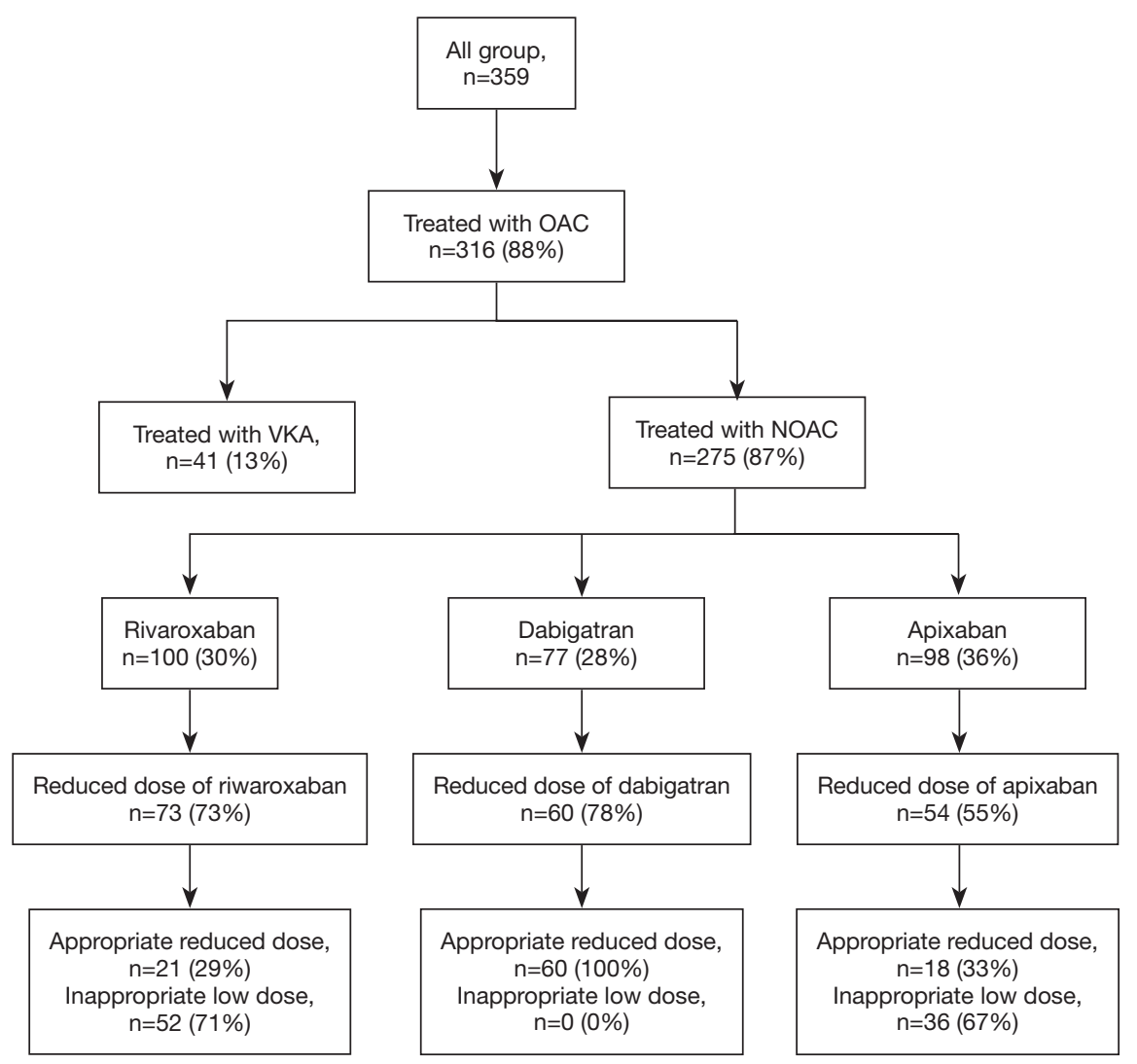

Figure 4 Analysis of oral anticoagulation therapy in the study group.

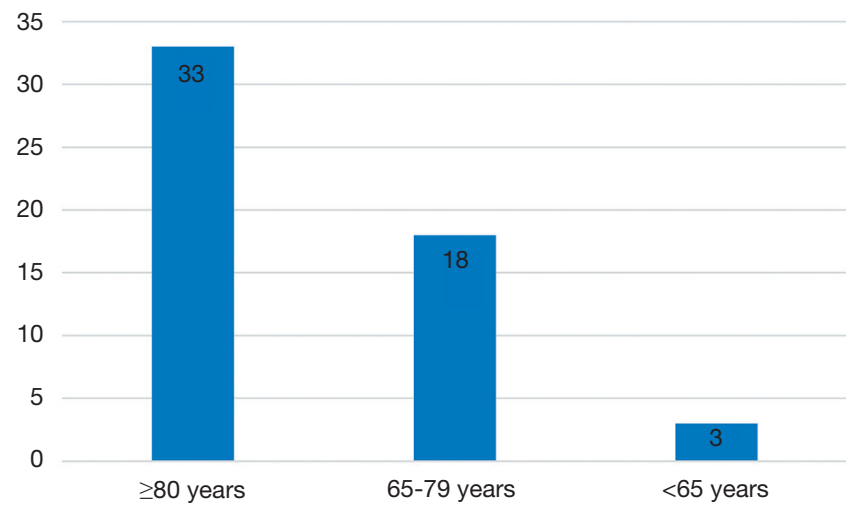

Figure 5 Age-dependency analysis of inappropriate low dose of apixaban.

efficacy compared to VKAs.

The studies mentioned above show that the advantage of using NOAC in combination antithrombotic therapy is mainly due to a lower frequency of bleeding complications.

Another interesting point that could potentially influence the prognosis, is the dose adjustment of NOAC.
All types of NOAC require dose adjustments based on certain clinical criteria. Notably, in most of the published RCTs, the dose of NOACs was reduced as recommended by FDA/EMA. In the PIONEER-AF trial, rivaroxaban was reduced from 15 to $10 \mathrm{mg}$ for patients with decreased creatinine clearance. Additionally, in the ENTRUST trial, edoxaban was reduced if moderate or severe renal impairment was observed. Finally, in the AUGUSTUS trial, the dose of apixaban was reduced according to the recommendations (depending on age, weight, and creatinine level). The treatment of such patients is challenging in terms of obtaining benefits while reducing complications. In our registry, we observed examples of both over- and undertreatment. In the case of dabigatran, a full dose was used in $21 \%$ of people with indications for its reduction. However, no patient was treated with reduced dabigatran without substantiation. On the other hand, underdosing was noticeable for apixaban and rivaroxaban (in $71 \%$ and $67 \%$ patients, respectively). Underdosing apixaban/rivaroxaban in our study is evidently more frequent than in other reports. In the study by Steinberg 
Table 4 The detailed characteristics of the inappropriate low dose group compared to the appropriate low dose group of patients treated with rivaroxaban and apixaban

\begin{tabular}{|c|c|c|c|}
\hline Characteristics & $\begin{array}{l}\text { Appropriate low dose NOAC } \\
\text { (rivaroxaban/apixaban); } n=39\end{array}$ & $\begin{array}{l}\text { Inappropriate low dose NOAC } \\
\text { (rivaroxaban/apixaban); } \mathrm{n}=88\end{array}$ & $P$ \\
\hline \multicolumn{4}{|l|}{ Demographic data } \\
\hline Female sex, n (\%) & $14(36 \%)$ & $34(39 \%)$ & 0.769 \\
\hline Heart failure & $29(74 \%)$ & $55(63 \%)$ & 0.193 \\
\hline HFrEF & $14(37 \%)$ & $28(33 \%)$ & 0.642 \\
\hline Valvular AF & $2(5 \%)$ & $2(2 \%)$ & 0.395 \\
\hline Ischemic stroke & $1(3 \%)$ & $15(17 \%)$ & 0.023 \\
\hline Peripheral embolism & $0(0 \%)$ & $0(0 \%)$ & - \\
\hline History of myocardial infarction & $24(62 \%)$ & $48(55 \%)$ & 0.463 \\
\hline History of CABG & $6(15 \%)$ & $10(11 \%)$ & 0.529 \\
\hline Previous $\mathrm{PCl}$ & $27(69 \%)$ & $49(56 \%)$ & 0.151 \\
\hline $\begin{array}{l}\text { Previous bleeding (including haemorrhagic stroke } \\
\text { and gastrointestinal bleeding) }\end{array}$ & $0(0 \%)$ & $3(3 \%)$ & 0.243 \\
\hline Thrombophilia & $0(0 \%)$ & $0(0 \%)$ & - \\
\hline Chronic obstructive pulmonary disease & $3(8 \%)$ & $9(10 \%)$ & 0.652 \\
\hline Asthma & $1(3 \%)$ & $2(2 \%)$ & 0.921 \\
\hline Hypothyroidism & $8(20 \%)$ & $9(10 \%)$ & 0.116 \\
\hline Hyperthyroidism & $2(5 \%)$ & $7(8 \%)$ & 0.567 \\
\hline Hypertension & $34(87 \%)$ & $77(87 \%)$ & 0.960 \\
\hline Chronic liver disease & $1(3 \%)$ & $4(5 \%)$ & 0.596 \\
\hline Alcohol abuse & $0(0 \%)$ & $6(7 \%)$ & 0.089 \\
\hline Antiplatelet therapy (ASA or clopidogrel) & $38(97 \%)$ & $83(94 \%)$ & 0.445 \\
\hline Antiplatelet therapy (ASA and clopidogrel) & $32(82 \%)$ & $78(88 \%)$ & 0.314 \\
\hline
\end{tabular}

Table 4 (continued) 
Table 4 (continued)

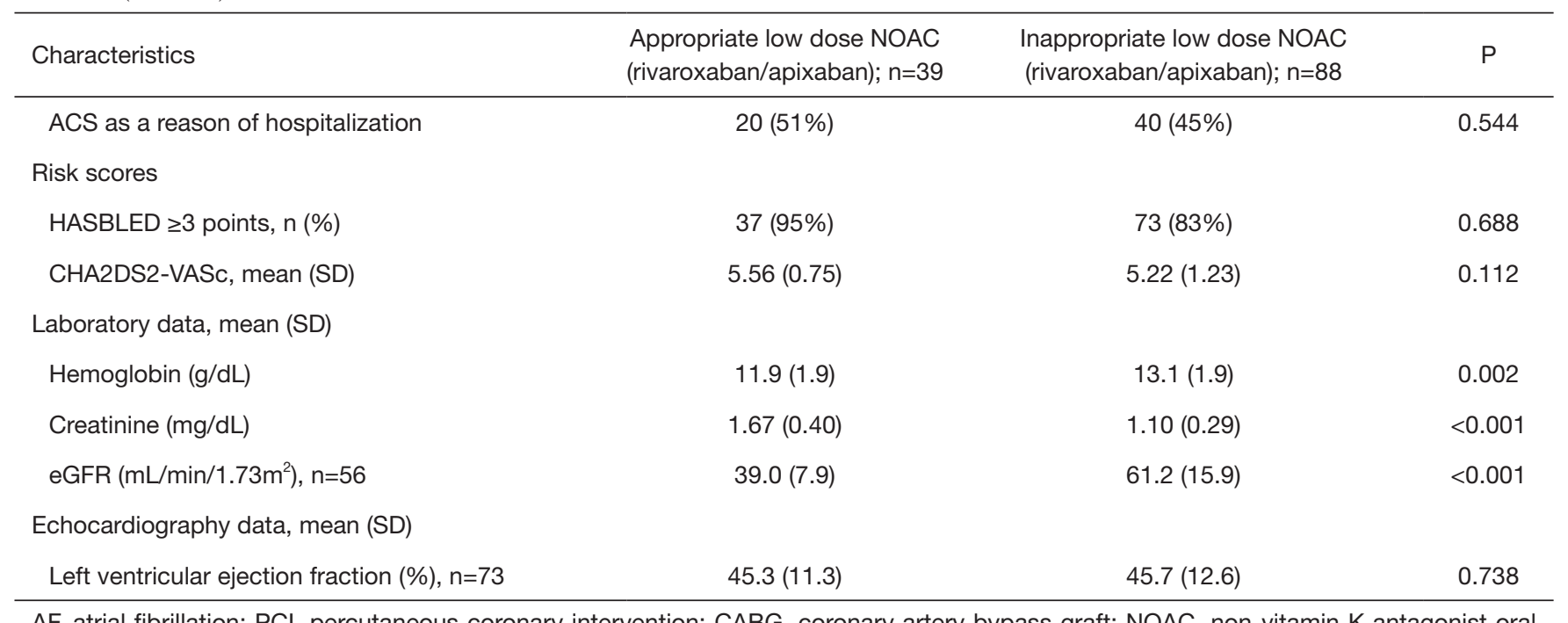

$\mathrm{AF}$, atrial fibrillation; $\mathrm{PCI}$, percutaneous coronary intervention; CABG, coronary artery bypass graft; NOAC, non-vitamin $\mathrm{K}$ antagonist oral anticoagulant; ASA, acetylsalicylic acid.

et al. (26), only $9.4 \%$ of patients were underdosed, $3.4 \%$ were overdosed, and all had an increased risk of cardiovascular events. In a single-study registry by Sato et al. (27), 23\% of AF patients were treated with inappropriate low doses of NOAC, and clinical factors such as age and creatinine clearance identified patients at risk of underdosed NOAC. In our study, the factors predisposing to using an inappropriate low dose of NOAC were mostly comparable to those shown in the studies mentioned above. In the case of age, kidney function, and body mass index, the most probable explanation is that reduced doses of rivaroxaban and apixaban are used even if the cut-offs for these parameters, which are mentioned as indication for a lower dose, are not reached. An exemplary analysis showing the dependence of an inappropriately low dose of apixaban on age depicts this phenomenon-a reduced dose was most frequently used in the elderly [even with a mass over $60 \mathrm{~kg}$ and creatinine below $1.5 \mathrm{mg} / \mathrm{dL}(133 \mu \mathrm{mol} / \mathrm{L})]$ than in slightly younger (65-79 years) subjects. Other authors also revealed the problem with using anticoagulation therapy in the patients with chronic kidney disease (28). In their study, OAC was less frequent used in the patients with kidney disease (defined as eGFR $\leq 60 \mathrm{~mL} / \mathrm{min} / 1.73 \mathrm{~m}^{2}$ ), even although the higher $\mathrm{CHA}_{2} \mathrm{DS}_{2} \mathrm{VASc}$ score. Moreover, in our analysis, an inappropriate low dose of NOAC was much more frequently used in patients after an ischemic stroke. We can only hypothesize that some clinical features, such as neurological defects and a higher risk of falling, may prompt the reduction of OAC in those subjects. The explanation for higher hemoglobin concentration as a factor predisposing to inappropriately low doses of NOAC is unclear and is probably a secondary statistical effect related to other factors discriminating both groups, with no clinical meaning.

\section{Strengths and limitations}

Our prospective multicenter registry shows real-life therapeutic decisions made in AF patients undergoing PCI in many Polish departments. It assesses their compliance with the guidelines and thus reveals the practices that should be carefully verified; however, it also has several limitations. First, we do not have detailed information about the periprocedural characteristics with respect to the coronary anatomy and PCI techniques, which limits the possibility of assessing the compliance of used antithrombotic therapy with the current guidelines. Second, due to the lack of longterm follow-up, it was not possible to assess long-term complications that may result from the applied treatment. Finally, we realized that the group of our patients is quite small and the larger study is needed to check the results obtained in our "pilot study".

\section{Conclusions}

In patients with $\mathrm{AF}$ undergoing $\mathrm{PCI}, \mathrm{NOACs}$ are definitely 
preferred over VKAs in TAT/DAT and an aggressive antithrombotic strategy with TAT is frequently chosen even if DAT is permissible by the guidelines. Label adherence of a reduced NOAC dose during combination therapy is not satisfactory for apixaban and rivaroxaban and probably results from too cautious an approach to the known indications for reduced therapy.

\section{Acknowledgments}

The POL-AF registry was initiated on the Scientific Platform of the "Club 30" of the Polish Cardiac Society. Investigators other than those listed as Authors include: Monika Budnik (Warsaw), Katarzyna Karoń (Warsaw), Monika Szewczak (Warsaw), Bartosz Krzemiński (Grodzisk Mazowiecki), Wiktor Wójcik (Warsaw).

Funding: None.

\section{Footnote}

Reporting Checklist: The authors have completed the STROBE reporting checklist. Available at: http://dx.doi. org/10.21037/cdt-20-839

Data Sharing Statement: Available at: http://dx.doi. org/10.21037/cdt-20-839

Conflicts of Interest: All authors have completed the ICMJE uniform disclosure form (available at http://dx.doi. org/10.21037/cdt-20-839). IG reports personal fees from Bayer and Boehringer-Ingelheim; AKC reports honoraria for lectures from Bayer, outside the submitted work; ATK reports research grant from Boehringer-Ingelheim, consultant for Boehringer-Ingelheim, Bayer, speaker for Boehringer-Ingelheim; BWK reports personal fees from Boehringer-Ingelheim, Bayer, Pfizer, outside the submitted work. The other authors have no conflicts of interest to declare.

Ethical Statement: The authors are accountable for all aspects of the work in ensuring that questions related to the accuracy or integrity of any part of the work are appropriately investigated and resolved. The study was conducted according to Good Clinical Practice guidelines and the Declaration of Helsinki (as revised in 2013). The study protocol was approved by the Ethics Committee of the Swietokrzyska Medical Chamber in Kielce (104/2018).
The Ethics Committee waived the requirement of obtaining informed consent from the patients to participate in the study.

Open Access Statement: This is an Open Access article distributed in accordance with the Creative Commons Attribution-NonCommercial-NoDerivs 4.0 International License (CC BY-NC-ND 4.0), which permits the noncommercial replication and distribution of the article with the strict proviso that no changes or edits are made and the original work is properly cited (including links to both the formal publication through the relevant DOI and the license). See: https://creativecommons.org/licenses/by-nc-nd/4.0/.

\section{References}

1. Kralev S, Schneider K, Lang S, et al. Incidence and severity of coronary artery disease in patients with atrial fibrillation undergoing first-time coronary angiography. PLoS One 2011;6:e24964.

2. Nieuwlaat R, Capucci A, Camm AJ, et al. Atrial fibrillation management: A prospective survey in ESC member countries: The euro heart survey on atrial fibrillation. Eur Heart J 2005;26:2422-34.

3. Nabauer M, Gerth A, Limbourg T, et al. The registry of the German competence NETwork on atrial fibrillation: Patient characteristics and initial management. Europace 2009;11:423-34.

4. Angiolillo DJ, Goodman SG, Bhatt DL, et al. Antithrombotic therapy in patients with atrial fibrillation undergoing percutaneous coronary intervention. A North American perspective-2016 update. Circ Cardiovasc Interv 2016;9:e004395.

5. Gibson CM, Mehran R, Bode Ch, et al. Prevention of bleeding in patients with atrial fibrillation undergoing PCI. N Engl J Med 2016;375:2423-34.

6. Dewilde WJ, Oirbans T, Verheugt FW, et al. Use of clopidogrel with or without aspirin in patients taking oral anticoagulant therapy and undergoing percutaneous coronary intervention: An open-label, randomised, controlled trial. Lancet 2013;381:1107-15.

7. Cannon CP, Bhatt DL, Oldgren J, et al. Dual antithrombotic therapy with dabigatran after PCI in atrial fibrillation. N Engl J Med 2017;377:1513-24.

8. Vranckx P, Lewalter T, Valgimigli M, et al. Evaluation of the safety and efficacy of an edoxaban-based antithrombotic regimen in patients with atrial 
fibrillation following successful percutaneous coronary intervention (PCI) with stent placement: Rationale and design of the ENTRUST-AF PCI trial. Am Heart J 2018;196:105-12.

9. Lopes RD, Vora AN, Liaw D, et al. An Open-label, 2 × 2 factorial, randomized controlled trial to evaluate the safety of apixaban vs. vitamin $\mathrm{K}$ antagonist and aspirin vs. placebo in patients with atrial fibrillation and acute coronary syndrome and/or percutaneous coronary intervention: Rationale and design of the AUGUSTUS trial. Am Heart J 2018;200:17-23.

10. Neumann FJ, Sousa-Uva M, Ahlsson A, et al 2018 ESC/ EACTS guidelines on myocardial revascularization. Eur Heart J 2019;40:87-165.

11. Valgimigli M, Bueno H, Byrne RA, et al. 2017 ESC Focused Update on Dual Antiplatelet Therapy in Coronary Artery Disease Developed in Collaboration with EACTS: The Task Force for Dual Antiplatelet Therapy in Coronary Artery Disease of the European Society of Cardiology (ESC) and of the European Association for Cardio-Thoracic Surgery (EACTS). Eur Heart J 2018;39:213-60.

12. Wang X, Fang L, Liu B, et al. Real-world comparisons of reduced-dose non-vitamin $\mathrm{K}$ antagonist oral anticoagulants versus warfarin in atrial fibrillation: a systematic review and meta-analysis. Heart Fail Rev 2020;25:973-83.

13. Lip GYH, Nieuwlaat R, Pisters R, et al. Refining clinical risk stratification for predicting stroke and thromboembolism in atrial fibrillation using a novel risk factor-based approach: The Euro heart survey on atrial fibrillation. Chest 2010;137:263-72.

14. Pisters R, Lane DA, Nieuwlaat R, et al. A novel userfriendly score (HAS-BLED) to assess 1-year risk of major bleeding in patients with atrial fibrillation: The Euro heart survey. Chest 2010;138:1093-100.

15. Knuuti J, Ijns W, Saraste A, et al 2019 ESC guidelines for the diagnosis and management of chronic coronary syndromes. The task force for the diagnosis and management of chronic coronary syndromes of the European society of cardiology (ESC). Eur Heart J 2020;41:407-77.

16. Hindricks G, Potpara T, Dagres N, et al. 2020 ESC guidelines for the diagnosis and management of atrial fibrillation developed in collaboration with the European Association of Cardio-Thoracic Surgery (EACTS). Eur Heart J 2020; ehaa612.

17. "Pradaxa," European Medicines Agency, last updated June
6, 2020. Available online: https://www.ema.europa.eu/en/ medicines/human/EPAR/pradaxa

18. "Xarelto," European Medicines Agency, last updated January 17, 2020. Available online: https://www.ema. europa.eu/en/medicines/human/EPAR/xarelto

19. "Eliquis," European Medicines Agency, last updated May 19, 2020. Available online: https://www.ema.europa.eu/en/ medicines/human/EPAR/eliquis

20. Uziębło-Życzkowska B, Krzesiński P, Jurek A, et al. Prevalence and risk factors of left atrial thrombus in patients with atrial fibrillation and lower class (IIa) recommendation to anticoagulants. Cardiovasc Diagn Ther 2020;10:717-24.

21. Bogacki P, Kabłak-Ziembicka A, Bryniarski K, et al. Triple anticoagulation therapy in patients with atrial fibrillation undergoing percutaneous coronary intervention - Real life assessment. Postepy Kardiol Interwencyjnej 2016;12:303-13.

22. D'Ascenzo F, Taha S, Moretti C, et al. Metaanalysis of randomized controlled trials and adjusted observational results of use of clopidogrel, aspirin, and oral anticoagulants in patients undergoing percutaneous coronary intervention. Am J Cardiol 2015;115:1185-93.

23. Eyileten C, Postula M, Jakubik D, et al. Non-vitamin K oral anticoagulants (NOAC) versus vitamin $\mathrm{k}$ antagonists (VKA) for atrial fibrillation with elective or urgent percutaneous coronary intervention: A meta-analysis with a particular focus on combination type. J Clin Med 2020;9:1120.

24. Lopes RD, Hong H, Harskamp RE, et al. Safety and efficacy of antithrombotic strategies in patients with atrial fibrillation undergoing percutaneous coronary intervention: A network meta-analysis of randomized controlled trials. JAMA Cardiol 2019;4:747-55.

25. Chi G, Kerneis M, Kalayci A, et al. Safety and efficacy of non-vitamin $\mathrm{K}$ oral anticoagulant for atrial fibrillation patients after percutaneous coronary intervention: A bivariate analysis of the PIONEER AF-PCI and REDUAL PCI trial. Am Heart J 2018;203:17-24.

26. Steinberg BA, Shrader P, Thomas L, et al. Off-label dosing of non-vitamin $\mathrm{K}$ antagonist oral anticoagulants and adverse outcomes: The ORBIT-AF II registry. J Am Coll Cardiol 2016;68:2597-604.

27. Sato T, Aizawa Y, Fuse K, et al. The comparison of inappropriate-low-doses use among 4 direct oral anticoagulants in patients with atrial fibrillation: From the database of a single-center registry. J Stroke Cerebrovasc 
Dis 2018;27:3280-8.

28. Tomaszuk-Kazberuk A, Nikas D, Lopatowska P, et al. Patients with Atrial Fibrillation and Chronic Kidney

Cite this article as: Uziębło-Życzkowska B, Krzesiński P, Maciorowska M, Gorczyca I, Jelonek O, Wójcik M, Błaszczyk R, Kapłon-Cieślicka A, Gawałko M, Tokarek T, RajtarSalwa R, Bil J, Wojewódzki M, Szpotowicz A, Krzciuk M, Bednarski J, Bakuła-Ostalska E, Tomaszuk-Kazberuk A, Szyszkowska A, Wełnicki M, Mamcarz A, Wożakowska-Kapłon B. Antithrombotic therapy in patients with atrial fibrillation undergoing percutaneous coronary intervention, including compliance with current guidelines-data from the POLish Atrial Fibrillation (POL-AF) Registry. Cardiovasc Diagn Ther 2021;11(1):14-27. doi: 10.21037/cdt-20-839
Disease More Often Undergo Angioplasty of Left Main Coronary Artery - a 867 Patient Study. Kidney Blood Press Res 2018;43:1796-805. 